\title{
Sudden Unexpected Death from Extraventricular Neurocytoma. A Case Report and Review of the Literature
}

\author{
Panagiotis Mylonakis ${ }^{1}$, Stefanos Milias ${ }^{2}$, Dimitrios Pappas ${ }^{3}$ and Antigony Mitselou ${ }^{4 *}$ \\ ${ }^{1}$ Medical Examiner's Office of Thessaloniki, Greece \\ ${ }^{2}$ Department of Pathology, 424 Military Hospital of Thessaloniki, Greece \\ ${ }^{3}$ Department of Pathology, 401 Military Hospital of Athens, Greece \\ ${ }^{4}$ Department of Forensic Pathology and Toxicology, University of Ioannina, Greece
}

Submission: April 06, 2017; Published: April 19, 2017

*Corresponding author: Panagiotis Mylonakis, MD, Medical Examiner's Office of Thessaloniki, Thessaloniki 54012, P.0.BOX: 19757, Greece, Tel: +030 2310550 416; Email: panaotis@yahoo.com

\begin{abstract}
Fatal brain tumors are often diagnosed well before death. Rarely, they are associated to sudden and unexpected death and encountered in medico legal autopsy practice. Neurocytomas are unusual neuronal tumors especially affecting young people and commonly arise in the ventricles with a benign outcome. Currently, these tumors have been well recognized outside the limits of the cerebral ventricules and in these instances, have been called "exta ventricular neurocytomas" (EVNs). The authors present the case of a 35 year-old male who died suddenly and unexpectedly due to a previously undiagnosed extra ventricular neurocytoma.
\end{abstract}

Keywords: Brain tumors; Neurocytoma; Extra ventricular neurocytoma; Sudden death; Autopsy

\section{Introduction}

Central neurocytomas (CNs) are benign tumors which usually arise from the lateral ventricles [1,2]. Extra ventricular neurocytomas (EVNs) refer to tumors with similar or identical biological and histopathological characteristics to CNs, but which arise from extra ventricular parenchymal tissue [2]. EVNs may occur at any age, but are usually seen in the third decade of life without sex preference [3]. Presenting symptoms obviously vary depending on the location of the tumor [4]. Histo pathologically, EVNs display a wide spectrum in morphology, such as the growth of monotonous neurocytes either in sheets, clusters, ribbons or rosettes, and neuropils dispersed in broad zones and contain a greater number of ganglion or ganglioid cells and astrocytes than CNs [5]. Furthermore, EVNs are often misdiagnosed as oligodendrogliomas, oligoastrocytomas or oligodendroglial tumors with neurocytic differentiation [6]. Immuno histo chemically, synaptophysin expression is positive and has been recognized as the most reliable diagnostic marker for identifying neuronal differentiation in brain neoplasia [7], and may thus play a vital role in the diagnosis of EVN tumors. Below we describe a rare case of sudden and unexpected death due to a clinically undiagnosed extra ventricular neurocytoma located on the midbrain.

\section{Case Report}

\section{History}

A well-developed, well-nourished, 35 year-old male was found dead, lying on the floor of his living-room and dressed up in his pyjamas. The investigation concluded that death occurred approximately 02:00 AM, of the same day his body was fount. His previous medical history did not include any significant signs or symptoms, besides a couple of syncoptic episodes within the last few months which were attributed to fatigue. A medico-legal autopsy was carried out to determine the cause of death.

\section{Autopsy findings}

The autopsy findings included congestion of the head and cyanosis of the bucal mucosa. Froth was evident around the mouth and nostrils. The skull bones and dura matter were normal without congenital or traumatic lesions. The brain weighed $1470 \mathrm{~g}$ and cerebral edema was evident. An almost spherical in shape mass lesion was found arising on the posterior surface 
of the midbrain, pressing against the pons and the cerebellum. The mass measured $1.5 \times 1.2 \times 1.0 \mathrm{~cm}$, and was well-defined, lobulated, grayish and soft (Figure 1). The heart weighted $380 \mathrm{~g}$ without coronary or myocardial lesions. The lungs were congestive and edematous. The right lung weighted $800 \mathrm{~g}$ and the left $860 \mathrm{~g}$. The rest of the internal organs were moderately congested, without any other pathology. Blood and urine were subjected to toxicological analysis which was negative for illegal drugs, medications and alcohol. Death was attributed to sudden cardiopulmonary arrest due to the tumor's pressure on the pons and cerebellum and was classified as natural.

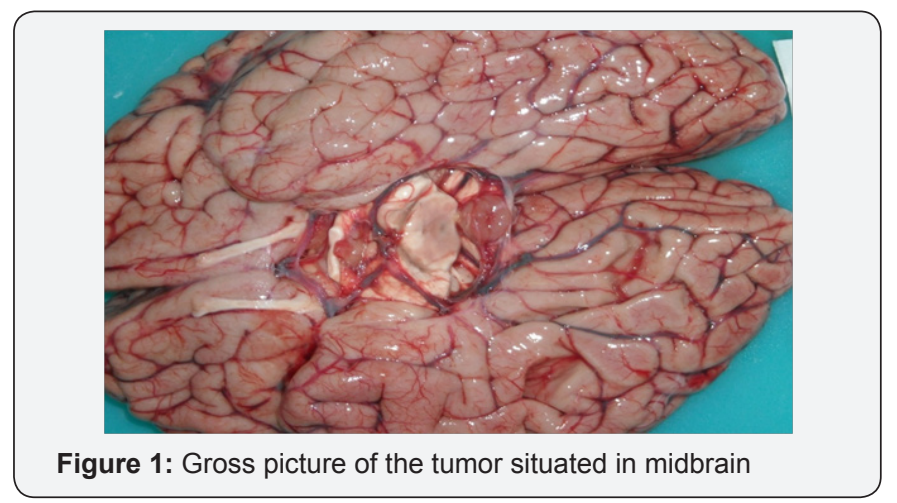

\section{Histologic findings and immune histo chemistry}

The tumor was fixed in $10 \%$ buffered neural formalin, entirely sampled, and was later embedded in paraffin. Some sections were stained with hematoxylin and eosin (H-E) for the morphological evaluation, whereas other sections were mounted on electrostatic slides and used for immune histo chemical study. Microscopically, the tumor consisted of uniform population of small, round cells with perinuclear halos, and small hyperchromatic nuclei with finely speckled chromatin (Figure 2). These cells were arranged in sheets and rarely formed rosettes, surrounding islands of fibrillary neuropil-like material. Rarely, ganglion cells were noted and appeared to be part of the tumor. A loose myxoid stroma was present and there was evidence of focal epithelial proliferation (Figures $3 \& 4$ ).

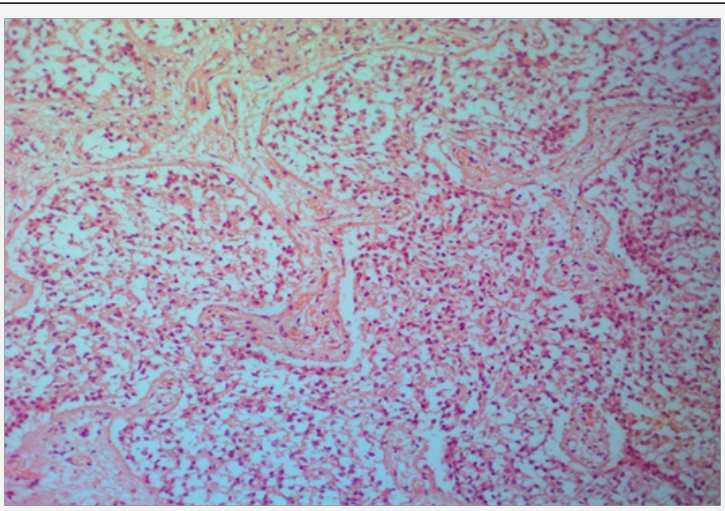

Figure 2: Microscopic characteristic of the tumor. Note clusters of tumor cells with neuropil islands (Hematoxylin and Eosin stain $\mathrm{x} 100)$.
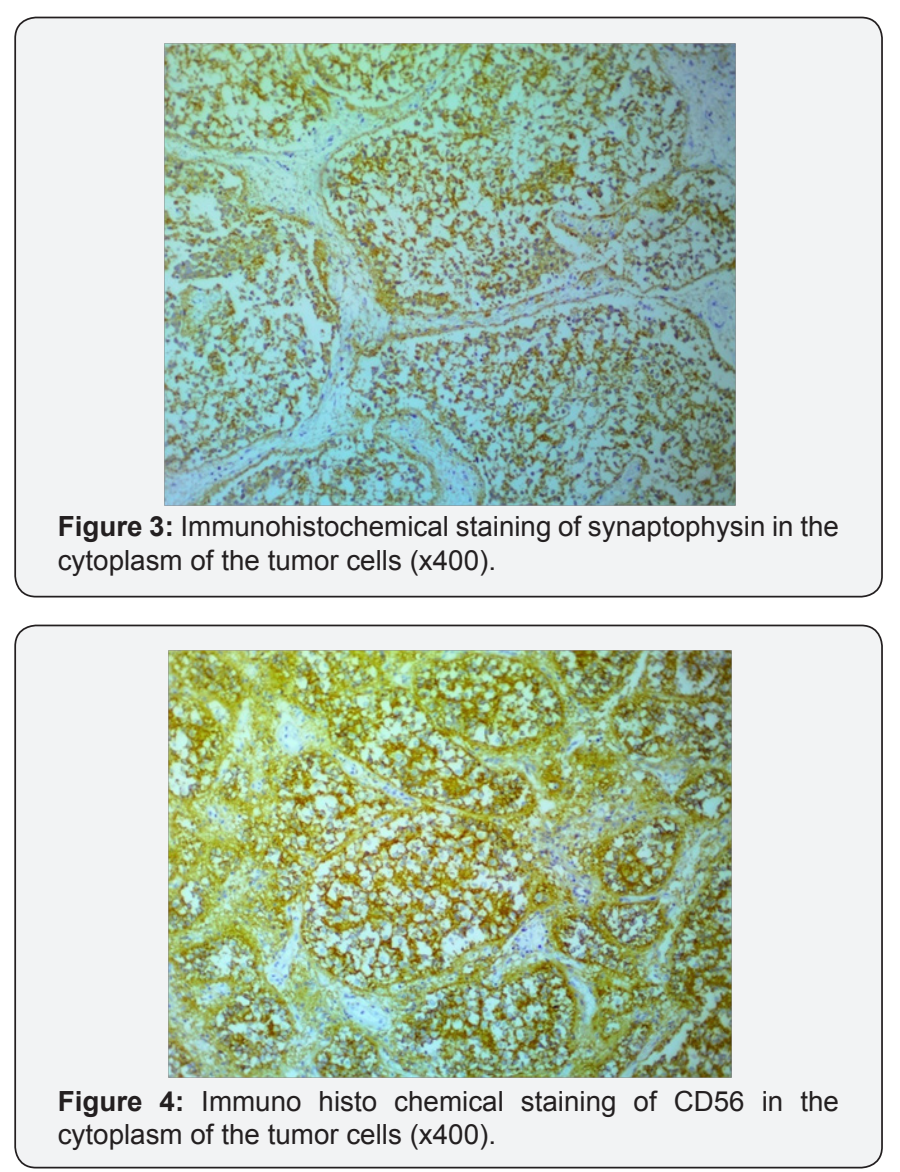

The primary used antibodies for immune histo chemistry were as follows: monoclonal antibodies against synaptophysin (SY), neuron specific enolase (NSE), glial fibrillary acidic protein (GFAP), CD56 and Ki-67 (MIB1); polyclonal antibodies against neurofilaments (NF), S-100 protein, chromogranin A (Chr A) epithelial membrane antigen (EMA), cytokeratines AE1/AE3 and $8 / 18$. The sections used for immune histo chemistry were air-dried overnight at $37 \mathrm{oC}$, deparaffinized through xylene, and dehydrated through graded alcohol. Endogenous peroxydase activity was blocked by immersing the specimens in a solution of $0.5 \% \mathrm{H} 2 \mathrm{O} 2$. To recover antigenicity, the slides were placed in a citrate buffer (10 mM, pH 6.0) and submitted to microwave antigen enhancement. The sections were allowed to cool down to room temperature, washed with phosphate-buffered saline solution (PBS, pH 7.4) and treated with normal horse serum to reduce non-specific antibody binding. After washing in PBS, the sections were incubated with biotinylated anti-mouse IgG and then with streptavidin-biotin-peroxydase complex reagent. Following extensive washing with PBS, the slides were treated with 3,3'diamino benzidine-hydrogen peroxide as a final indicator, and lightly counterstained with Harri's hematoxylin.

\section{Results}

Immuno histo chemical results led to diffuse and intense staining for SY, NF, CD56, and NSE in the cytoplasm, disclosing the neuronal nature of the lesion; S-100 protein, keratins AE1/ 
AE3and 8/18, chromogranin A, and EMA were negative. GFAP expression was also negative, with only some reactive astrocytes that were weakly positive. The proliferation marker MIB1 gave evidence of a very low growth fraction $(<1 \%)$.

\section{Discussion}

Sudden unexpected deaths from occult primary intracranial neoplasms in any location are rare. Reported frequencies have ranged from $0.02 \%$ to $2.1 \%$ in medico-legal autopsy series $[8,9]$. However, reviewing these reports reveals that the majority of individuals, have had clinical symptoms of an intracranial process, often of long duration, before death [10]. Symptoms like headache, seizures, nonspecific cognitive of personality changes, or focal neurologic signs are reported, and their intensity depends on tumor's grade, development rate and localization. Thus, these deaths may be sudden but cannot be regarded as unexpected. Furthermore, most of the subjects have survived for some time after the acute event, and the number of those who have been found dead or who have died instantaneously is indeed small. Mortality due to brain neoplasm is particularly high in young adults aged 20 to 39 years and the overall incidence rate was 10.47 per 100,000 populations between 2008 to 2012 [11].

However, some intracranial tumors, usually glial, can occasionally be asymptomatic. Actually, the most frequent intracranial neoplasm causing sudden death is glioblastma, while astrocytoma grade II is also presented with an increased incidence [8,9]. Other non symptomatic brain tumors, with a very low incidence, reported to cause sudden death may be benign or malignant [12]. The benign reported tumors included colloid cysts [9], meningiomas, pilocytic astrocytomas, ependymomas, lipomas [13], subependymomas, teratomas, gangliogliomas, schwannomas, hemangioblastomas, and central neurocytomas. In addition, malignant or high grade brain tumors like anaplastic astrocytoma, medulloblastoma, malignant lymphoma, germinoma, gliosarcoma, malignant glioma, and anaplastic ependymoma were also reported [12]. So nowadays taking in consideration the characteristic symptoms of a brain tumor and the widely use of current imaging techniques (Computed Tomography, Magnetic Resonance Image, Positron Emission Tomography), the occurrence of sudden and unexpected death caused by a primary tumor is an uncommon, or rather rare phenomenon [14].

Central neurocytoma was first described by Hassoun et al in 1982, as a tumor of neuronal origin in the central nervous system (CNS) [1], comprising $0.2 \%-0.5 \%$ of all CNS tumors $[1,15]$. Originally thought to be exclusively intra ventricular, Ferroel et al. and Mishio et al. first reported unusual cases of extra ventricular neurocytoma in 1989 [2]. EVN was described in the WHO classification of CNS tumors as an oligodendrogliomamimicking tumor [16]. Historically, many such lesions were regarded as oligodendrogliomas until detailed immune cyto chemical classification of their neuronal phenotype was established. With only about 100 cases reported in the literature so far, the exact incidence of EVN is unclear and still rare. Liu et al. reported an incidence of $0.13 \%$ in their series of brain tumors [17].

Typically, EVN affects young adults around the third decade, with cases ranging from 5 to 76 years of age (median 34 years), with no gender predilection [2]. EVNs have been reported from almost every site in the nervous system but the most common site is the frontal lobe, followed by the parietal, temporal and occipital lobes [2,5]. EVNs are also found at unusual intracranial locations like the thalamus and hypothalamus [5], cerebellum [18], pons [19,20], sellar region [21], pineal gland [4], base of the skull [4], and spinal cord [4] as well. Kane et al. in their meta-analysis study, observed the frontal lobe $(31.8 \%)$ to be the commonest site, followed by temporal $(20 \%)$ and parietal lobes $(15.3 \%)$ [21]. The average size of the tumor was measured $4.3 \mathrm{~cm}$ as the maximum diameter [21].

Extra ventricular neurocytoma of the midbrain or brainstem is very rare, and only two cases has been published so far [19, 20]. In the first report, the authors described the case of an 18-year-old male who was found to have a $1.2 \mathrm{~cm}$ solid mass in the right pons in the region of the superior cerebellar pedicle, which was totally resected via a right suboccipital craniotomy approach. After excision the patient was alive and well, with no recurrence of the tumor [19]. The second case was a 58 year-old man with a $2.6 \times 2.2 \mathrm{~cm}$ ring enhancing cystic mass in the right pons demonstrated by MRI scans. A stereotactic biopsy of the mass was performed and the final pathological diagnosis was atypical extra ventricular neurocytoma. The MIB1 tumor LI was $6 \%$ to $8 \%$. The patient was initially treated beam radiotherapy and then chemotherapy because of tumor progression, but his symptoms worsened. The patient underwent a right suboccipital craniotomy with aggressive subtotal resection of the tumor. The patient 28 months after surgery was alive and no evidence of residual or recurrent tumor was found in MRI scans [20].

The most common clinical presentation is related to raised intracranial pressure, but may vary depending on tumor location from seizure to gait disturbances. The onset of symptoms may vary from three months to ten years and the average duration of clinical symptoms and signs was 32.5 months [4]. EVN resulting in an acute or sub-acute presentation is extremely uncommon and there has been no forensic autopsy case report. We have found only two case reports of central intra ventricular neurocytomas associated with sudden unexpected death of an adult in which the diagnosis was not established before death $[22,23]$. In our case, we investigated an extra ventricular neurocytoma without major symptoms, till the day of sudden and unexpected death, as the first report in the literature. Grossly, neurocytomas are generally grayish in color, soft in consistency, resembling the gray matter, well circumscribed and lobulated, with varying calcifications and occasional haemorrhage $[2,15]$. 
Microscopically, neurocytomas are well-differentiated tumors with benign histology, which may display varied histological architecture even in the same specimen $[2,15]$. The honeycomb arrangement of oligodendroglioma, and large fibrillary areas resembling irregular rosettes of pineocytoma are the commonest patterns identified in central neurocytoma [2]. In other areas, cells may be arranged in straight lines or as perivascular rosettes mimicking ependymoma. These tumors are composed of uniform, small-to-medium-sized cell with rounded nuclei, finely stippled chromatine ('salt and pepper" chromatin) and inconspicuous nucleoli, along with scant cytoplasm $[2,4,7]$. Cells are usually apposed but may also be set within a background of finely filamentous stroma having a neuropil-like quality.

Hematoxylin and eosin (H-E) stained sections often reveals fixation artifacts in the form of cytoplasmic vacuolations, closely resembling the 'fried-egg' appearance of oligodendroglioma. Vascularity is represented by long, thin walled capillary-sized vessels, which are arranged in a linear arborizing pattern, imparting an endocrine appearance. In many cases, thin-walled dilated channels, and foci of calcification are readily indentified. In places, tumor display dense cellular areas altering with fibrillar/ acellular areas. The later components are mainly peri vascular and have a fine fibrillary neuropil matrix mimicking 'rosettes of ependymoma'. Calcifications in the form of small calcospherites distributed throughout the tumor parenchyma have been noted in half of biopsies while rare cases exhibit features such as Homer-Wright rosettes, neuroblastic component, or ganglioid cell $[2,4,7]$.

Typical extra ventricular neurocytomas display a wide morphological spectrum encompassing arrangement of neurocytes in sheet, clusters, ribbons, or rosettes, with neuropil dispersed either in broad zones (neuropil islands) between these cells arrangements or localized within the rosettes [2,4,7]. Neurocytes most often demonstrates finely granular, slightly eosinophilic cytoplasm, occasionally with artefactual vacuolations resembling oligodendroglioma. Nuclei of these tumors are striking monotonous and rounded, with finely speckled chromatin, distinct nuclear rims, and one to three nucleoli. Ganglionic differentiation is more common in EVN and has been observed in $66 \%$ cases in one series [5-7] These were either focal (29\%) or diffuse (37\%) with few cases showing compact nodules of ganglion cells against a neurocytic background [5-7]. Mitotic activity is uncommon while calcification is frequent. Cerebral EVNs generally have pulsing along well-defined interface with adjacent brain parenchyma [57].

Differential diagnosis includes oligodendroglioma, ependymoma, dysembryoblastic neuroepithelial tumor, medullocytomas or lipidized medulloblastomas, and cerebellar liponeurocytoma [2,4]. However, the tumor most commonly considered in the differential diagnosis of neurocytoma is oligodendroglioma. There are some differences that should be considered to the pathological diagnosis. The delicate vascular pattern with a 'chicken wire' aspect, the presence of minigemistocytes and perineural satellitosis is more characteristic of oligodendroglioma; on the other hand, a predominant fibrillary background is more typical of neurocytoma $[2,4]$.

Almost all neurocytomas and neurocytoma-like lesions have been diagnosed on the basis of synaptophysin immunoreactivity alone $[2,7]$. Strong immunostaining for synaptophysin have been recognized as the most suitable and reliable diagnostic marker. Typically, synaptophysin immunoreactivity is noted in the neuropil, especially in fibrillary zones and perivascular cell-free areas, and not in the cell bodies of normal neurons. Tumor cells have been reported to express an array of neuronal markers, including neuron-specific enolase (NSE), neuron-associated class II beta-tubulin, tau, microtubule-associated protein-2 (MAP2), calcineurin, protein gene product 9,5 (PGP9.5), HNK1 (leu 7), neuron-specific antigen II, and embryonal form of neuronal cell adhesion molecule (ECAM) [2,24]. Neuronal nuclear antigen (NeuN) expression is generally associated with tumor cells displaying terminal neuronal differentiation and is often helpful in resolving ambiguous synaptophysin staining. Interestingly, $\mathrm{Hu}$-immunoreactivity is restricted to the nuclei of neurocytes only, especially in those surrounding neuropil islands. Chromogranin A and neurofilaments are usually absent, except when admixed with ganglion cells [2,24].

Expression of GFAP has been observed more frequently in EVN compared with CN. It may be difficult to distinguish GFAPimmuno reactive and entrapped non neo plastic astrocytes from tumor cells showing focal GFAP immune staining, with neurocytic features [25]. Through this bears testimony to origin from bi potential cells of germinal matrix, this distinction appears to bear little or no prognostic connotation [24]. In our case, the tumor presented to diffuse and intense staining for SY, NF, CD56, and NSE in the cytoplasm, disclosing the neuronal nature of the lesion; S-100 protein, keratins AE1/AE3and 8/18, chromogranin A, and EMA were negative. GFAP expression was also negative, with only some reactive astrocytes that were weakly positive. The proliferation marker MIB1 gave evidence of a very low growth fraction $(<1 \%)$.

Most neurocytomas are cytologically bland and mitotically deprived and correspond to WHO grade II [16]. Occasional cases, however, display histological features of anaplasia or malignancy without clinical evidence of poor outcome $[2,4,15]$, for which Mackenzie et al. reserved the designation "proliferating neurocytoma" [26]. Typically, proliferative index of neurocytomas as evaluated by silver colloidal staining for argyrophilic nucleolar organizer regions (AgNOR counts) or by Ki-67 and proliferating cell nuclear antigen (PCNA) immune labeling have been low [27]. Currently, MIB-1 labeling index (MIB-1 LI) is rutenly used to assess proliferation potential of neurocytomas [28]. In our case the Ki-67 LI was $<1 \%$. 
The terms "atypical neurocytoma" / "atypical extra ventricular neurocytoma" have been coined for CN/EVN exhibiting MIB-1LI >2\% with/without anaplastic features like focal necrosis, vascular proliferation, and increased mitotic activity. Cases with MIB-1 LI >2\% have been noted to predict adverse outcome in terms of time of recurrence or progression after surgery [20,28].

Molecular, genetic abnormalities of EVN have not been well characterized until the present. Although some authors have reported $1 \mathrm{p} / 19 \mathrm{q}$ co-deletion in EVN, these reports are limited by the small number of involved cases and lack of isocitrate dehydrogenase enzyme isoform (IDH1 R132) and 2 (IDH2 R 172) mutation studies [29]. Recently, Rodriguez et al. reported that the $1 p / 19 q$ co-deletion was seen in approximately $25 \%$ of their 21 cases of EVN, associated with aggressive histological features [30].

In conclusion, the autopsy of the descendant presented in this article revealed the presence of a primary neoplasm of the midbrain, an extra ventricular neurocytoma demonstrated by histology and immune histo chemistry, and we believe it was directly responsible for causing sudden cardiopulmonary arrest and death. The existence of the tumor was previously undiagnosed clinically, thus the patient's death should be regarded as "sudden" and "unexpected".

\section{References}

1. Hassoun J, Gambarelli D, Grisoli F, Pellet W, Salomon G, et al. (1982) Central neurocytoma. A electron-microscopic study of two cases. Acta Neuropathol 56(2): 151-156.

2. Sharma MC, Dep P, Sharma S, Sarkar C (2006) Neurocytoma: a comprehensive review. Neurosurg Rev 29(4): 270-285.

3. Figueiredo EG, Welling L, Rosemberg S, Paiva W, Teixeira MJ (2010) Extraventricular neurocytomas. BMJ Case Reports 1-3.

4. Patil AS, Menon G, Easwer HV, Nair S (2014) Extra ventricular neurocytoma, a comprehensive review. Acta Neurochir 156(2): 349354.

5. Brat DJ, Scheithauer BW, Eberhart CG, Burger PC (2001) Extraventricular neurocytomas. Pathologic features and clinical outcome. Am J Surg Pathol 25(10): 1252-1260.

6. Bureger PC, Scherhauer BW, Vogel FS (2002) Neuronal, glioneural and neurocytic tumors. In: Burger PC, Scherhauer BW, et al. (Eds.), Surgical pathology of the nervous system and its coverings. ( $4^{\text {th }}$ edn), Churchill Livingstone, New York, pp. 264-291.

7. Robbins P, Segal A, Norula S (1995) Central neurocytoma. A clinicopathological, immunohistochemical and ultrastructural study of 7 cases. Pathol Res Pract 191(2): 100-111.

8. Vougiouklakis T, Mitselou A, Agnantis NJ (2006) Sudden death due to primary intracranial neoplasms. A forensic autopsy study. Anticancer Res 26(3): 2463-2466.

9. DiMaio SM, DiMaio VI, Kirkpatrik JB (1980) Sudden unexpected death due to primary intracranial neoplasms. Am J Forensic Med Pathol 1(1): $29-45$.

10. Lindboe CF, Svenes KB, Slordal L (1997) Sudden unexpected death in subjects with undiagnosed gliomas. Am J Forensic Med Pathol 18(3): 271-275.
11. Ostrom QT, Gitlemann H, Falop J, Liu M, Bland R, et al. (2015) CBTRUS Statistical Report: Primary brain and central nervous system tumours diagnosed in the United States in 2008-2012. Neuro-Oncology 17(4): 1-62.

12. Matschke J, Tokos M (2005) Sudden unexpected death due to undiagnosed glioblastoma-report of three cases and review of the literature. Int J Legal Med 119: 280-284.

13. Siritt JV, Mitselou A, Tovar M, Viloria J (1986) Lipoma of the brain in a pregnant woman as a cause of death. A case report. Patol Hum (Vzla) 11: 197-199.

14. Eberhart ChG, Morrison A, Gyure KA, Frazier J, Smialek JE, et al. (2001) Decreasing incidence of sudden death due to undiagnosed primary central nervous system. Arch Pathol Lab Med 125(8): 1024-1030.

15. Hassoun J, Soylemezoglu F, Gambarelli D, Figarella-Branger D, von Ammon K, et al. (1993) Central neurocytoma: a synopsis of clinical and histological features. Brain Pathol 3(3): 297-306.

16. Louis DN, Ohgaki H, Wistler OD (2007) The 2007 WHO classification of tumours of the central nervous system. Acta Neuropathol 114: 97-109.

17. Liu K, Wen G, Lv X, Deng Y, Hou G, et al. (2013) MR imaging of cerebral extra ventricular neurocytoma: a reporte of 9 cases. AJNR. AM J Neuroradiol 34: 541-546.

18. Enam S, Resenblum M, Ho K (1997) Neurocytoma in the cerebellum. J Neurosurg 87(1): 100-102.

19. Soontornniyomkj FV, Schelper RI (1996) Pontine neurocytoma. J Clin Pathol 49: 764-765.

20. Swinson BM, friedman WA, Yachnis AT (2006) Pontine atypical neurocytoma: a case report. Neurosurgery 58(5): 990-997.

21. Kane AJ, Sughrue ME, Rutkowski MJ (2012) Atypia predicting prognosis for intracranial extra ventricular neurocytomas. J Neurosurg 116(2): 349-354.

22. Balko MG, Schultz DL (1999) Sudden death due to a central neurocytoma. Am J Forensic Med Pathol 20(2): 180-183.

23. Gill J, Koelmeyer TD (2009) Death in custody and undiagnosed central neurocytoma. Am J Forensic Med Pathol 30(3): 289-291.

24. You H, Kim YL, Im SY, Sub-Kim H, Park SH, et al. (2005) Immunohistochemical study of central neurocytoma, subependymoma, and subependymal gian cell astrocytoma. J Neurooncol 74(1): 1-8.

25. Xiong Z, Zhang J, Li Z, Atan Q Sun S, et al. (2015) A comparative study of intra ventricular central neurocytomas and extra ventricular neurocytomas. J Neuroonvol 121(3): 521-529.

26. Mackenzie IR (1999) Central neurocytoma: histologic atypia, proliferation potential, and clinical outcome. Cancer 85(7): 1606-1661.

27. Hara A, Anki Y, Shineda J, Hirayama H, Nikawa S, et al. (1993) Central neurocytoma: proliferative assessment by nucleolar organizer region staining. Surg Neurol 39(5): 343-347.

28. Kim DG, Kim JS, Chi JG, Park SH, Jung HW, et al. (1996) Central neurocytomas: proliferative potential and biological behavior. J Neurosurg 84(5): 742-747.

29. Myung JK, Cho HJ, Park CK, Chung CK, Choi SH, et al. (2013) Clinicopathological and genetic characteristics of extraventricular neurocytomas. Neuropathology 33(2): 111-121.

30. Rodriguez FJ, Mota RA, Scheitauer BW (2009) Intephase cytogenetics for $1 \mathrm{p} 19 \mathrm{q}$ and $\mathrm{t}(1 ; 19)(\mathrm{q} 10 ; \mathrm{p} 10)$ may distinguish prognostically relevant subgroups in extra ventricular neurocytoma. Brain Pathol 19: 623-629. 
(C) Cors This work icensed under Creative (C) BY DoI: $10.19080 /$ JFSCI.2017.03.555603

\section{Your next submission with Juniper Publishers will reach you the below assets}

- Quality Editorial service

- Swift Peer Review

- Reprints availability

- E-prints Service

- Manuscript Podcast for convenient understanding

- Global attainment for your research

- Manuscript accessibility in different formats ( Pdf, E-pub, Full Text, Audio)

- Unceasing customer service

Track the below URL for one-step submission https://juniperpublishers.com/online-submission.php 